\title{
Ground state homoclinic orbits of superquadratic damped vibration systems
}

\section{Guanwei Chen ${ }^{1 *}$ and Xiaoming Zhao ${ }^{2}$}

\section{*Correspondence:}

guanweic@163.com

'School of Mathematics and

Statistics, Anyang Normal University,

Anyang, Henan Province 455000,

P.R. China

Full list of author information is

available at the end of the article

\begin{abstract}
In the case where nonlinearities are superquadratic at infinity, we study the existence of ground state homoclinic orbits for damped vibration systems without periodic conditions by using variational methods. Here the (local) Ambrosetti-Rabinowitz superquadratic condition is replaced by a general superquadratic condition.

MSC: $49 \mathrm{~J} 40 ; 70 \mathrm{H} 05$
\end{abstract}

Keywords: damped vibration systems; ground state homoclinic orbits; superlinear; variant weak linking theorem

\section{Introduction and main result}

We shall study the existence of ground state homoclinic orbits for the following damped vibration system:

$$
\ddot{u}(t)+M \dot{u}(t)-L(t) u(t)+H_{u}(t, u(t))=0, \quad t \in \mathbb{R},
$$

where $M$ is an antisymmetric $N \times N$ constant matrix, $L(t) \in C\left(\mathbb{R}, \mathbb{R}^{N \times N}\right)$ is a symmetric matrix, $H(t, u) \in C^{1}\left(\mathbb{R} \times \mathbb{R}^{N}, \mathbb{R}\right)$ and $H_{u}(t, u)$ denotes its gradient with respect to the $u$ variable. We say that a solution $u(t)$ of (1.1) is homoclinic (to 0$)$ if $u(t) \in C^{2}\left(\mathbb{R}, \mathbb{R}^{N}\right.$ ) such that $u(t) \rightarrow 0$ and $\dot{u}(t) \rightarrow 0$ as $|t| \rightarrow \infty$. If $u(t) \not \equiv 0$, then $u(t)$ is called a nontrivial homoclinic solution.

If $M=0$ (zero matrix), then (1.1) reduces to the following second order Hamiltonian system:

$$
\ddot{u}(t)-L(t) u(t)+H_{u}(t, u(t))=0, \quad t \in \mathbb{R} .
$$

This is a classical equation which can describe many mechanic systems, such as a pendulum. In the past decades, the existence and multiplicity of periodic solutions and homoclinic orbits for (1.2) have been studied by many authors via variational methods; see [1-17] and the references therein.

The periodicity assumption is very important in the study of homoclinic orbits for (1.2) since periodicity is used to control the lack of compactness due to the fact that (1.2) is set on all $\mathbb{R}$. However, non-periodic problems are quite different from the ones described in periodic cases. Rabinowitz and Tanaka [10] introduced a type of coercive condition on the 
matrix $L(t)$ :

$$
l(t):=\inf _{|u|=1}(L(t) u, u) \rightarrow+\infty \quad \text { as }|t| \rightarrow \infty
$$

and obtained the existence of homoclinic orbit for non-periodic (1.2) under the Ambrosetti-Rabinowitz (AR) superquadratic condition:

$$
0<\mu H(t, u) \leq\left(H_{u}(t, u), u\right), \quad \forall t \in \mathbb{R}, \forall u \in \mathbb{R}^{N} \backslash\{0\},
$$

where $\mu>2$ is a constant, $(\cdot, \cdot)$ denotes the standard inner product in $\mathbb{R}^{N}$ and the associated norm is denoted by $|\cdot|$.

We should mention that the case where $M \neq 0$, i.e., the damped vibration system (1.1), only a few authors have studied homoclinic orbits of (1.1); see [18-22]. Zhu [22] considered the periodic case of (1.1) (i.e., $L(t)$ and $H(t, u)$ are $T$-periodic in $t$ with $T>0$ ) and obtained the existence of nontrivial homoclinic solutions of (1.1). The authors [18-21] considered the non-periodic case of (1.1): Zhang and Yuan [21] obtained the existence of at least one homoclinic orbit for (1.1) when $H$ satisfies the subquadratic condition at infinity by using a standard minimizing argument; By a symmetric mountain pass theorem and a generalized mountain pass theorem, Wu and Zhang [20] obtained the existence and multiplicity of homoclinic orbits for (1.1) when $H$ satisfies the local $(A R)$ superquadratic growth condition:

$$
0<\mu H(t, u) \leq\left(H_{u}(t, u), u\right), \quad \forall t \in \mathbb{R}, \forall|u| \geq r
$$

where $\mu>2$ and $r>0$ are two constants. Notice that the authors [20,21] all used the condition (1.3). Recently, Chen $[18,19]$ obtained infinitely many homoclinic orbits for $(1.1)$ when $H$ satisfies the subquadratic [18] and asymptotically quadratic [19] condition at infinity by the following weaker conditions than (1.3):

$\left(\mathrm{L}_{1}\right)$ There is a constant $\beta>1$ such that

$$
\operatorname{meas}\left\{t \in \mathbb{R}:|t|^{-\beta} L(t)<b I_{N}\right\}<+\infty, \quad \forall b>0 .
$$

$\left(\mathrm{L}_{2}\right)$ There is a constant $\gamma \geq 0$ such that

$$
l(t):=\inf _{|u|=1}(L(t) u, u) \geq-\gamma, \quad \forall t \in \mathbb{R},
$$

which were firstly used in [14]. It is not hard to check that the matrix-valued function $L(t):=\left(t^{4} \sin ^{2} t+1\right) I_{N}$ satisfying $\left(\mathrm{L}_{1}\right)$ and $\left(\mathrm{L}_{2}\right)$, but not satisfying $(1.3)$.

We define an operator $J: H^{1}\left(\mathbb{R}, \mathbb{R}^{N}\right) \rightarrow H^{1}\left(\mathbb{R}, \mathbb{R}^{N}\right)$ by

$$
(J u, v):=\int_{\mathbb{R}}(M u(t), \dot{v}(t)) d t, \quad \forall u, v \in H^{1}\left(\mathbb{R}, \mathbb{R}^{N}\right) .
$$

Since $M$ is an antisymmetric $N \times N$ constant matrix, $J$ is self-adjoint on $H^{1}\left(\mathbb{R}, \mathbb{R}^{N}\right)$. Let $\chi$ denote the self-adjoint extension of the operator $-\frac{d^{2}}{d t^{2}}+L(t)+J$. We are interested in the indefinite case:

$\left(\mathrm{J}_{1}\right) \sup (\sigma(\chi) \cap(-\infty, 0))<0<\inf (\sigma(\chi) \cap(0, \infty))$. 
Let $\widetilde{H}(t, u):=\frac{1}{2}\left(H_{u}(t, u), u\right)-H(t, u)$. We assume the following.

$\left(\mathrm{H}_{1}\right) \quad H(t, u) \in C^{1}\left(\mathbb{R} \times \mathbb{R}^{N}, \mathbb{R}\right)$ and $\left|H_{u}(t, u)\right|=o(|u|)$ as $|u| \rightarrow 0$ uniformly in $t$.

$\left(\mathrm{H}_{2}\right) \frac{H(t, u)}{|u|^{2}} \rightarrow+\infty$ as $|u| \rightarrow+\infty$ uniformly in $t$, and $H(t, u) \geq 0, \forall(t, u) \in \mathbb{R} \times \mathbb{R}^{N}$.

$\left(\mathrm{H}_{3}\right) \widetilde{H}(t, u)>0$ if $u \neq 0$, and for any $a, b>0$ we have

$$
\inf \left\{\frac{\widetilde{H}(t, u)}{|u|^{2}}: t \in \mathbb{R} \text { and } u \in \mathbb{R}^{N} \text { with } a \leq|u|<b\right\}>0 .
$$

$\left(\mathrm{H}_{4}\right)$ There are constants $c_{0}, r_{0}>0$ and $\sigma>1$ such that

$$
\frac{\left|H_{u}(t, u)\right|^{\sigma}}{|u|^{\sigma}} \leq c_{0} \widetilde{H}(t, u) \quad \text { if }|u| \geq r_{0}, \forall t \in \mathbb{R}
$$

Now, our main result reads as follows.

Theorem 1.1 If $\left(\mathrm{J}_{1}\right),\left(\mathrm{L}_{1}\right)-\left(\mathrm{L}_{2}\right)$, and $\left(\mathrm{H}_{1}\right)-\left(\mathrm{H}_{4}\right)$ hold, then $(1.1)$ has a ground state homoclinic orbit.

Remark 1.1 Although the authors [20] have studied the superquadratic case of (1.1), it is not hard to check that our superquadratic condition $\left(\mathrm{H}_{2}\right)$ is weaker than the condition (1.4) (see Example 1.1). Moreover, we obtain the existence of ground state homoclinic orbits of (1.1), i.e., nontrivial homoclinic orbits with least energy of the action functional of (1.1).

Example 1.1 Let

$$
H(t, u)=g(t)\left(|u|^{p}+(p-2)|u|^{p-\varepsilon} \sin ^{2}\left(|u|^{\varepsilon} / \varepsilon\right)\right)
$$

where $p>2,0<\varepsilon<p-2$ and $g(t)>0$ is continuous. It is not hard to check that $H$ satisfies $\left(\mathrm{H}_{1}\right)-\left(\mathrm{H}_{4}\right)$ but does not satisfy $(1.4)$.

The following abstract critical point theorem plays an important role in proving our main result. Let $W$ be a Hilbert space with norm $\|\cdot\|$ and have an orthogonal decomposition $W=N \oplus N^{\perp}, N \subset W$ is a closed and separable subspace. There exists a norm $|V|_{\omega}$ that satisfies $|v|_{\omega} \leq\|v\|$ for all $v \in N$ and induces a topology equivalent to the weak topology of $N$ on bounded subset of $N$. For $u=v+z \in W=N \oplus N^{\perp}$ with $v \in N, z \in N^{\perp}$, we define $|u|_{\omega}^{2}=|v|_{\omega}^{2}+\|z\|^{2}$. Particularly, if $\left(u_{n}=v_{n}+z_{n}\right)$ is $\|\cdot\|$-bounded and $u_{n} \stackrel{|\cdot| \omega_{\omega}}{\rightarrow} u$, then $v_{n} \rightarrow v$ weakly in $N, z_{n} \rightarrow z$ strongly in $N^{\perp}, u_{n} \rightarrow v+z$ weakly in $W$ (cf. [23]).

Let $W=W^{-} \oplus W^{+}, z_{0} \in W^{+}$with $\left\|z_{0}\right\|=1$. Let $N:=W^{-} \oplus \mathbb{R} z_{0}$ and $W_{1}^{+}:=N^{\perp}=$ $\left(W^{-} \oplus \mathbb{R} z_{0}\right)^{\perp}$. For $R>0$, let

$$
Q:=\left\{u:=u^{-}+s z_{0}: s \in \mathbb{R}^{+}, u^{-} \in W^{-},\|u\|<R\right\}
$$

with $P_{0}=s_{0} z_{0} \in Q, s_{0}>0$. We define

$$
D:=\left\{u:=s z_{0}+z^{+}: s \in \mathbb{R}, z^{+} \in W_{1}^{+},\left\|s z_{0}+z^{+}\right\|=s_{0}\right\} .
$$


For $\Phi \in C^{1}(W, \mathbb{R})$, we define

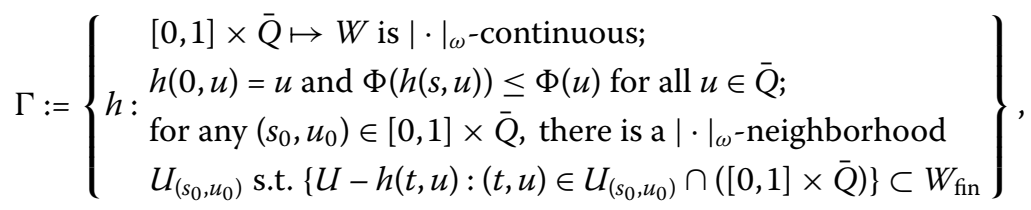

where $W_{\text {fin }}$ denotes various finite-dimensional subspaces of $W, \Gamma \neq 0$, since $i d \in \Gamma$.

We shall use the following variant weak linking theorem to prove our result.

Theorem A ([23]) The family of $C^{1}$-functional $\left\{\Phi_{\lambda}\right\}$ has the form

$$
\Phi_{\lambda}(u):=J(u)-\lambda K(u), \quad \forall \lambda \in[1,2]
$$

Assume that

(a) $K(u) \geq 0, \forall u \in W, \Phi_{1}=\Phi$;

(b) $J(u) \rightarrow \infty$ or $K(u) \rightarrow \infty$ as $\|u\| \rightarrow \infty$;

(c) $\Phi_{\lambda}$ is $|\cdot|_{\omega}$-upper semicontinuous, $\Phi_{\lambda}^{\prime}$ is weakly sequentially continuous on $W$. Moreover, $\Phi_{\lambda}$ maps bounded sets to bounded sets;

(d) $\sup _{\partial Q} \Phi_{\lambda}<\inf _{D} \Phi_{\lambda}, \forall \lambda \in[1,2]$.

Then for almost all $\lambda \in[1,2]$, there exists a sequence $\left\{u_{n}\right\}$ such that

$$
\sup _{n}\left\|u_{n}\right\|<\infty, \quad \Phi_{\lambda}^{\prime}\left(u_{n}\right) \rightarrow 0, \quad \Phi_{\lambda}\left(u_{n}\right) \rightarrow c_{\lambda}
$$

where $c_{\lambda}:=\inf _{h \in \Gamma} \sup _{u \in Q} \Phi_{\lambda}(h(1, u)) \in\left[\inf _{D} \Phi_{\lambda}, \sup _{\bar{Q}} \Phi\right]$

The rest of the present paper is organized as follows. In Section 2, we give some preliminary lemmas, which are useful in the proof of our main result. In Section 3, we give the detailed proof of our main result.

\section{Preliminaries}

In this section, we firstly give the variational frameworks of our problem and some related preliminary lemmas, and then give the detailed proof of the main result.

In the following, we use $\|\cdot\|_{p}$ to denote the norm of $L^{p}\left(\mathbb{R}, \mathbb{R}^{N}\right)$ for any $p \in[1, \infty]$. Let $E:=H^{1}\left(\mathbb{R}, \mathbb{R}^{N}\right)$ be a Hilbert space with the inner product and norm given, respectively, by

$$
\langle u, v\rangle_{E}=\int_{\mathbb{R}}[(\dot{u}(t), \dot{v}(t))+(u(t), v(t))] d t, \quad\|u\|_{E}=\langle u, u\rangle_{E}^{1 / 2}, \quad \forall u, v \in E .
$$

It is well known that $E$ is continuously embedded in $L^{p}\left(\mathbb{R}, \mathbb{R}^{N}\right)$ for $p \in[2, \infty)$. We define an operator $J: E \rightarrow E$ by

$$
(J u, v):=\int_{\mathbb{R}}(M u(t), \dot{v}(t)) d t, \quad \forall u, v \in E .
$$

Since $M$ is an antisymmetric $N \times N$ constant matrix, $J$ is self-adjoint on $E$. Moreover, we denote by $\chi$ the self-adjoint extension of the operator $-\frac{d^{2}}{d t^{2}}+L(t)+J$ with the domain $\mathcal{D}(\chi) \subset L^{2}\left(\mathbb{R}, \mathbb{R}^{N}\right)$. 
Let $W:=\mathcal{D}\left(|\chi|^{1 / 2}\right)$, the domain of $|\chi|^{1 / 2}$. We define, respectively, on $W$ the inner product and the norm

$$
\langle u, v\rangle_{W}:=\left(|\chi|^{1 / 2} u,|\chi|^{1 / 2} v\right)_{2}+(u, v)_{2} \quad \text { and } \quad\|u\|_{W}=\langle u, u\rangle_{W}^{1 / 2}
$$

where $(\cdot, \cdot)_{2}$ denotes the inner product in $L^{2}\left(\mathbb{R}, \mathbb{R}^{N}\right)$.

By a similar proof of Lemma 3.1 in [14], we can prove the following lemma.

Lemma 2.1 If conditions $\left(\mathrm{L}_{1}\right)$ and $\left(\mathrm{L}_{2}\right)$ hold, then $W$ is compactly embedded into $L^{p}\left(\mathbb{R}, \mathbb{R}^{N}\right)$ for all $1 \leq p \leq+\infty$.

By Lemma 2.1, it is easy to prove that the spectrum $\sigma(\chi)$ has a sequence of eigenvalues (counted with their multiplicities)

$$
\lambda_{1} \leq \lambda_{2} \leq \cdots \leq \lambda_{k} \leq \cdots \rightarrow \infty
$$

and the corresponding system of eigenfunctions $\left\{e_{k}: k \in \mathbb{N}\right\}\left(\chi e_{k}=\lambda_{k} e_{k}\right)$ forms an orthogonal basis in $L^{2}\left(\mathbb{R}, \mathbb{R}^{N}\right)$.

By $\left(\mathrm{J}_{1}\right)$, we may let

$$
k_{1}:=\sharp\left\{j: \lambda_{j}<0\right\}, \quad W^{-}:=\operatorname{span}\left\{e_{1}, \ldots, e_{k_{1}}\right\}, \quad W^{+}:=\operatorname{cl}_{W}\left(\operatorname{span}\left\{e_{k_{1}}, \ldots\right\}\right) .
$$

Then one has the orthogonal decomposition

$$
W=W^{-} \oplus W^{+}
$$

with respect to the inner product $\langle\cdot, \cdot\rangle_{W}$. Now, we introduce, respectively, on $W$ the following new inner product and norm:

$$
\langle u, v\rangle:=\left(|\chi|^{1 / 2} u,|\chi|^{1 / 2} v\right)_{2}, \quad\|u\|=\langle u, u\rangle^{1 / 2},
$$

where $u, v \in W=W^{-} \oplus W^{+}$with $u=u^{-}+u^{+}$and $v=v^{-}+v^{+}$. Clearly, the norms $\|\cdot\|$ and $\|\cdot\|_{W}$ are equivalent (see [3]), and the decomposition $W=W^{-} \oplus W^{+}$is also orthogonal with respect to both inner products $\langle\cdot, \cdot\rangle$ and $(\cdot, \cdot)_{2}$.

For problem (1.1), we consider the following functional:

$$
\Phi(u)=\frac{1}{2} \int_{\mathbb{R}}\left[|\dot{u}(t)|^{2}+(M u(t), \dot{u}(t))+(L(t) u(t), u(t))\right] d t-\int_{\mathbb{R}} H(t, u) d t, \quad u \in W .
$$

Then $\Phi$ can be rewritten as

$$
\Phi(u)=\frac{1}{2}\left\|u^{+}\right\|^{2}-\frac{1}{2}\left\|u^{-}\right\|^{2}-\int_{\mathbb{R}} H(t, u) d t, \quad u=u^{-}+u^{+} \in W .
$$

Let $I(u):=\int_{\mathbb{R}} H(t, u) d t$. In view of the assumptions of $H$, we know $\Phi, I \in C^{1}(W, \mathbb{R})$ and the derivatives are given by

$$
I^{\prime}(u) v=\int_{\mathbb{R}}\left(H_{u}(t, u), v\right) d t, \quad \Phi^{\prime}(u) v=\left\langle u^{+}, v^{+}\right\rangle-\left\langle u^{-}, v^{-}\right\rangle-I^{\prime}(u) v,
$$

for any $u, v \in W=W^{-} \oplus W^{+}$with $u=u^{-}+u^{+}$and $v=v^{-}+v^{+}$. By the discussion of [24], the (weak) solutions of system (1.1) are the critical points of the $C^{1}$ functional $\Phi: W \rightarrow \mathbb{R}$. 
Moreover, it is easy to verify that if $u \not \equiv 0$ is a solution of (1.1), then $u(t) \rightarrow 0$ and $\dot{u}(t) \rightarrow 0$ as $|t| \rightarrow \infty$ (see Lemma 3.1 in [25]).

In order to apply Theorem A, we consider

$$
\Phi_{\lambda}(u):=\frac{1}{2}\left\|u^{+}\right\|^{2}-\lambda\left(\frac{1}{2}\left\|u^{-}\right\|^{2}+\int_{\mathbb{R}} H(t, u) d t\right) .
$$

It is easy to see that $\Phi_{\lambda}$ satisfies conditions (a), (b) in Theorem A. To see (c), if $u_{n} \stackrel{|\cdot| \omega}{\rightarrow} u$, then $u_{n}^{+} \rightarrow u^{+}$and $u_{n}^{-} \rightarrow u^{-}$in $W$, going to a subsequence if necessary, $u_{n} \rightarrow u$ a.e. on $\mathbb{R}$. By Fatou's lemma and the weak lower semicontinuity of the norm, we have

$$
\varlimsup_{n \rightarrow \infty} \Phi_{\lambda}\left(u_{n}\right) \leq \Phi_{\lambda}(u)
$$

which means that $\Phi_{\lambda}$ is $|\cdot|_{\omega}$-upper semicontinuous. $\Phi_{\lambda}^{\prime}$ is weakly sequentially continuous on $W$ is due to [26]. To continue the discussion, we still need to verify condition (d) in Theorem A.

Lemma 2.2 Under the assumptions of Theorem 1.1, we have the following facts:

(i) There exists $\rho>0$ independent of $\lambda \in[1,2]$ such that $\kappa:=\inf \Phi_{\lambda}\left(S_{\rho} W^{+}\right)>0$, where

$$
S_{\rho} W^{+}:=\left\{z \in W^{+}:\|z\|=\rho\right\} .
$$

(ii) For fixed $z_{0} \in W^{+}$with $\left\|z_{0}\right\|=1$ and any $\lambda \in[1,2]$, there is $R>\rho>0$ such that $\sup \Phi_{\lambda}(\partial Q) \leq 0$, where $Q:=\left\{u:=u^{-}+s z_{0}: s \in \mathbb{R}^{+}, u^{-} \in W^{-},\|u\|<R\right\}$.

Proof (i) Under assumptions $\left(\mathrm{H}_{1}\right)$ and $\left(\mathrm{H}_{4}\right)$, we know for any $\varepsilon>0$ there exists $C_{\varepsilon}>0$ such that

$$
\left|H_{u}(t, u)\right| \leq \varepsilon|u|+C_{\varepsilon}|u|^{p-1}
$$

and

$$
|H(t, u)| \leq \varepsilon|u|^{2}+C_{\varepsilon}|u|^{p},
$$

where $p \geq \frac{2 \sigma}{\sigma-1}>2$ with $\sigma>1$. Hence, for any $u \in W^{+}$,

$$
\Phi_{\lambda}(u) \geq \frac{1}{2}\|u\|^{2}-\lambda \varepsilon\|u\|^{2}-C_{\varepsilon}^{\prime}\|u\|^{p},
$$

which implies the conclusion.

(ii) Suppose by contradiction that there exist $u_{n} \in W^{-} \oplus \mathbb{R}^{+} z_{0}$ such that $\Phi_{\lambda}\left(u_{n}\right)>0$ for all $n$ and $\left\|u_{n}\right\| \rightarrow \infty$ as $n \rightarrow \infty$. Let $v_{n}:=\frac{u_{n}}{\left\|u_{n}\right\|}=s_{n} z_{0}+v_{n}^{-}$, then

$$
0<\frac{\Phi_{\lambda}\left(u_{n}\right)}{\left\|u_{n}\right\|^{2}}=\frac{1}{2}\left(s_{n}^{2}-\lambda\left\|v_{n}^{-}\right\|^{2}\right)-\lambda \int_{\mathbb{R}} \frac{H\left(t, u_{n}\right)}{\left|u_{n}\right|^{2}}\left|v_{n}\right|^{2} d t .
$$

It follows from $H(t, u) \geq 0\left(\right.$ see $\left.\left(\mathrm{H}_{2}\right)\right)$ that

$$
\left\|v_{n}^{-}\right\|^{2} \leq \lambda\left\|v_{n}^{-}\right\|^{2}<s_{n}^{2}=1-\left\|v_{n}^{-}\right\|^{2},
$$

therefore, $\left\|v_{n}^{-}\right\| \leq \frac{1}{\sqrt{2}}$ and $1-\frac{1}{\sqrt{2}} \leq s_{n} \leq 1$. 
Thus $s_{n} \rightarrow s \neq 0$ after passing to a subsequence, $v_{n} \rightarrow v$ and $v_{n} \rightarrow v$ a.e. on $\mathbb{R}$. Hence, $v=s z_{0}+v^{-} \neq 0$ and, since $\left|v_{n}\right| \rightarrow \infty$ if $v \neq 0$, it follows from $\left(\mathrm{H}_{2}\right)$ and Fatou's lemma that

$$
\int_{\mathbb{R}} \frac{H\left(t, u_{n}\right)}{\left|u_{n}\right|^{2}}\left|v_{n}\right|^{2} d t \rightarrow+\infty
$$

contrary to (2.5). The proof is finished.

Applying Theorem A, we soon obtain the following facts.

Lemma 2.3 Under the assumptions of Theorem 1.1, for almost all $\lambda \in[1,2]$, there exists a sequence $\left\{u_{n}\right\}$ such that

$$
\sup _{n}\left\|u_{n}\right\|<\infty, \quad \Phi_{\lambda}^{\prime}\left(u_{n}\right) \rightarrow 0, \quad \Phi_{\lambda}\left(u_{n}\right) \rightarrow c_{\lambda} \in\left[\kappa, \sup _{\bar{Q}} \Phi\right] .
$$

Lemma 2.4 Under the assumptions of Theorem 1.1, for almost all $\lambda \in[1,2]$, there exists a $u_{\lambda}$ such that

$$
\Phi_{\lambda}^{\prime}\left(u_{\lambda}\right)=0, \quad \Phi_{\lambda}\left(u_{\lambda}\right) \leq \sup _{\bar{Q}} \Phi .
$$

Proof Let $\left\{u_{n}\right\}$ be the sequence obtained in Lemma 2.3, write $u_{n}=u_{n}^{-}+u_{n}^{+}$with $u_{n}^{ \pm} \in W^{ \pm}$. Since $\left\{u_{n}\right\}$ is bounded, $\left\{u_{n}^{+}\right\}$is also bounded, then $u_{n} \rightarrow u_{\lambda}$ and $u_{n}^{+} \rightarrow u_{\lambda}^{+}$in $W$, after passing to a subsequence.

We claim that $u_{\lambda}^{+} \neq 0$. If not, then Lemma 2.1 implies $u_{n}^{+} \rightarrow 0$ in $L^{q}\left(\mathbb{R}, \mathbb{R}^{N}\right)$ for all $q \in$ $[1,+\infty]$. It follows from the definition of $\Phi$, Hölder's inequality, and (2.3) that

$$
\begin{aligned}
0 & \leq \int_{\mathbb{R}}\left|\left(H_{u}\left(t, u_{n}\right), u_{n}^{+}\right)\right| d t \leq \varepsilon \int_{\mathbb{R}}\left|u_{n}\right| \cdot\left|u_{n}^{+}\right| d t+C_{\varepsilon} \int_{\mathbb{R}}\left|u_{n}\right|^{p-1}\left|u_{n}^{+}\right| d t \\
& \leq \varepsilon\left\|u_{n}\right\|_{2}\left\|u_{n}^{+}\right\|_{2}+C_{\varepsilon}\left\|u_{n}\right\|_{p}^{p-1}\left\|u_{n}^{+}\right\|_{p} \rightarrow 0 .
\end{aligned}
$$

It follows from (2.2) and Lemma 2.3 that

$$
\Phi_{\lambda}\left(u_{n}\right) \leq\left\|u_{n}^{+}\right\|^{2}=\Phi_{\lambda}^{\prime}\left(u_{n}\right) u_{n}^{+}+\lambda \int_{\mathbb{R}}\left(H_{u}\left(t, u_{n}\right), u_{n}^{+}\right) d t \rightarrow 0,
$$

which contradicts with the fact that $\Phi_{\lambda}\left(u_{n}\right) \geq \kappa$. Hence, $u_{\lambda}^{+} \neq 0$, and thus $u_{\lambda} \neq 0$. Note that $\Phi_{\lambda}^{\prime}$ is weakly sequentially continuous on $W$, thus

$$
\Phi_{\lambda}^{\prime}\left(u_{\lambda}\right) z=\lim _{n \rightarrow \infty} \Phi_{\lambda}^{\prime}\left(u_{n}\right) z=0, \quad \forall z \in W .
$$

By $\left(\mathrm{H}_{3}\right)$, Fatou's lemma, and Lemma 2.3, we have

$$
\begin{aligned}
\sup _{\bar{Q}} \Phi \geq c_{\lambda} & =\lim _{n \rightarrow \infty}\left(\Phi_{\lambda}\left(u_{n}\right)-\frac{1}{2} \Phi_{\lambda}^{\prime}\left(u_{n}\right) u_{n}\right) \\
& =\lim _{n \rightarrow \infty} \lambda \int_{\mathbb{R}}\left(\frac{1}{2}\left(H_{u}\left(t, u_{n}\right), u_{n}\right)-H\left(t, u_{n}\right)\right) d t \\
& \geq \lambda \int_{\mathbb{R}}\left(\frac{1}{2}\left(H_{u}\left(t, u_{\lambda}\right), u_{\lambda}\right)-H\left(t, u_{\lambda}\right)\right) d t=\Phi_{\lambda}\left(u_{\lambda}\right) .
\end{aligned}
$$

Thus we get $\Phi_{\lambda}\left(u_{\lambda}\right) \leq \sup _{\bar{Q}} \Phi$. 
Lemma 2.5 Under the assumptions of Theorem 1.1, there exists $\lambda_{n} \rightarrow 1$ and $\left\{u_{\lambda_{n}}\right\}$ such that

$$
\Phi_{\lambda_{n}}^{\prime}\left(u_{\lambda_{n}}\right)=0, \quad \Phi_{\lambda_{n}}\left(u_{\lambda_{n}}\right) \leq \sup _{\bar{Q}} \Phi
$$

Moreover, $\left\{u_{\lambda_{n}}\right\}$ is bounded.

Proof The existence of $\left\{u_{\lambda_{n}}\right\}$ such that

$$
\Phi_{\lambda_{n}}^{\prime}\left(u_{\lambda_{n}}\right)=0, \quad \Phi_{\lambda_{n}}\left(u_{\lambda_{n}}\right) \leq \sup _{\bar{Q}} \Phi
$$

is the direct consequence of Lemma 2.4. To prove the boundedness of $\left\{u_{\lambda_{n}}\right\}$, arguing by contradiction, suppose that $\left\|u_{\lambda_{n}}\right\| \rightarrow \infty$. Let $v_{\lambda_{n}}:=\frac{u_{\lambda_{n}}}{\left\|u_{\lambda_{n}}\right\|}$. Then $\left\|v_{\lambda_{n}}\right\|=1, v_{\lambda_{n}} \rightarrow v$ in $W$ and $v_{\lambda_{n}} \rightarrow v$ a.e. in $\mathbb{R}$, after passing to a subsequence.

Recall that $\Phi_{\lambda_{n}}^{\prime}\left(u_{\lambda_{n}}\right)=0$. Thus for any $\varphi \in W$, we have

$$
\left\langle u_{\lambda_{n}}^{+}, \varphi\right\rangle-\lambda_{n}\left\langle u_{\lambda_{n}}^{-}, \varphi\right\rangle=\lambda_{n} \int_{\mathbb{R}}\left(H_{u}\left(t, u_{\lambda_{n}}\right), \varphi\right) d t .
$$

Consequently $\left\{v_{\lambda_{n}}\right\}$ satisfies

$$
\left\langle v_{\lambda_{n}}^{+}, \varphi\right\rangle-\lambda_{n}\left\langle v_{\lambda_{n}}^{-}, \varphi\right\rangle=\lambda_{n} \int_{\mathbb{R}} \frac{\left(H_{u}\left(t, u_{\lambda_{n}}\right), \varphi\right)}{\left\|u_{\lambda_{n}}\right\|} d t
$$

Let $\varphi=v_{\lambda_{n}}^{ \pm}$in (2.7), respectively. Then we have

$$
\left\langle v_{\lambda_{n}}^{+}, v_{\lambda_{n}}^{+}\right\rangle=\lambda_{n} \int_{\mathbb{R}} \frac{\left(H_{u}\left(t, u_{\lambda_{n}}\right), v_{\lambda_{n}}^{+}\right)}{\left\|u_{\lambda_{n}}\right\|} d t
$$

and

$$
-\lambda_{n}\left\langle v_{\lambda_{n}}^{-}, v_{\lambda_{n}}^{-}\right)=\lambda_{n} \int_{\mathbb{R}} \frac{\left(H_{u}\left(t, u_{\lambda_{n}}\right), v_{\lambda_{n}}^{-}\right)}{\left\|u_{\lambda_{n}}\right\|} d t
$$

Since $1=\left\|v_{\lambda_{n}}\right\|^{2}=\left\|v_{\lambda_{n}}^{+}\right\|^{2}+\left\|v_{\lambda_{n}}^{-}\right\|^{2}$, we have

$$
1=\int_{\mathbb{R}} \frac{\left(H_{u}\left(t, u_{\lambda_{n}}\right), \lambda_{n} v_{\lambda_{n}}^{+}-v_{\lambda_{n}}^{-}\right)}{\left\|u_{\lambda_{n}}\right\|} d t
$$

For $r \geq 0$, let

$$
h(r):=\inf \left\{\widetilde{H}(t, u): t \in \mathbb{R} \text { and } u \in \mathbb{R}^{N} \text { with }|u| \geq r\right\} .
$$

By $\left(\mathrm{H}_{1}\right)$ and $\left(\mathrm{H}_{3}\right)$, we have $h(r)>0$ for all $r>0$. By $\left(\mathrm{H}_{3}\right)$ and $\left(\mathrm{H}_{4}\right)$, for $|u| \geq r_{0}$,

$$
c_{0} \tilde{H}(t, u) \geq \frac{\left|H_{u}(t, u)\right|^{\sigma}}{|u|^{\sigma}}=\left(\frac{\left|H_{u}(t, u)\right||u|}{|u|^{2}}\right)^{\sigma} \geq\left(\frac{\left(H_{u}(t, u), u\right)}{|u|^{2}}\right)^{\sigma} \geq\left(\frac{2 H(t, u)}{|u|^{2}}\right)^{\sigma},
$$

it follows from $\left(\mathrm{H}_{2}\right)$ and the definition of $h(r)$ that

$$
h(r) \rightarrow \infty \quad \text { as } r \rightarrow \infty .
$$


For $0<a<b$, let

$$
\Omega_{n}(a, b):=\left\{t \in \mathbb{R}: a \leq\left|u_{\lambda_{n}}(t)\right|<b\right\}
$$

and

$$
C_{a}^{b}:=\inf \left\{\frac{\widetilde{H}(t, u)}{|u|^{2}}: t \in \mathbb{R} \text { and } u \in \mathbb{R}^{N} \text { with } a \leq|u|<b\right\} .
$$

By $\left(\mathrm{H}_{3}\right)$, we have $C_{a}^{b}>0$ and

$$
\widetilde{H}\left(t, u_{\lambda_{n}}\right) \geq C_{a}^{b}\left|u_{\lambda_{n}}\right|^{2} \quad \text { for all } t \in \Omega_{n}(a, b) \text {. }
$$

Since $\Phi_{\lambda_{n}}^{\prime}\left(u_{\lambda_{n}}\right)=0$ and $\Phi_{\lambda_{n}}\left(u_{\lambda_{n}}\right) \leq \sup _{\bar{Q}} \Phi$, there exists a constant $C_{0}>0$ such that for all $n$

$$
C_{0} \geq \Phi_{\lambda_{n}}\left(u_{\lambda_{n}}\right)-\frac{1}{2} \Phi_{\lambda_{n}}^{\prime}\left(u_{\lambda_{n}}\right) u_{\lambda_{n}}=\int_{\mathbb{R}} \tilde{H}\left(t, u_{\lambda_{n}}\right) d t
$$

from which we have

$$
\begin{aligned}
C_{0} & \geq \int_{\Omega_{n}(0, a)} \widetilde{H}\left(t, u_{\lambda_{n}}\right) d t+\int_{\Omega_{n}(a, b)} \widetilde{H}\left(t, u_{\lambda_{n}}\right) d t+\int_{\Omega_{n}(b, \infty)} \widetilde{H}\left(t, u_{\lambda_{n}}\right) d t \\
& \geq \int_{\Omega_{n}(0, a)} \widetilde{H}\left(t, u_{\lambda_{n}}\right) d t+C_{a}^{b} \int_{\Omega_{n}(a, b)}\left|u_{\lambda_{n}}\right|^{2} d t+h(b)\left|\Omega_{n}(b, \infty)\right| .
\end{aligned}
$$

Invoking $\left(\mathrm{H}_{4}\right)$, set $\tau:=2 \sigma /(\sigma-1)$ and $\sigma^{\prime}=\tau / 2$. Since $\sigma>1$ one sees $\tau \in(2,+\infty)$. Fix arbitrarily $\hat{\tau} \in(\tau,+\infty)$. By (2.10) and the fact $h(r) \rightarrow \infty$ as $r \rightarrow \infty$, we have

$$
\left|\Omega_{n}(b, \infty)\right| \leq \frac{C_{0}}{h(b)} \rightarrow 0
$$

as $b \rightarrow \infty$ uniformly in $n$, it follows from $\left\|v_{\lambda_{n}}\right\|=1$, Hölder's inequality, and Sobolev's embedding theorem that

$$
\int_{\Omega_{n}(b, \infty)}\left|v_{\lambda_{n}}\right|^{\tau} d t \leq C\left|\Omega_{n}(b, \infty)\right|^{1-\frac{\tau}{\tau}} \rightarrow 0
$$

as $b \rightarrow \infty$ uniformly in $n$. By (2.10) and $\left\|u_{\lambda_{n}}\right\| \rightarrow \infty$, for any fixed $0<a<b$,

$$
\int_{\Omega_{n}(a, b)}\left|v_{\lambda_{n}}\right|^{2} d t=\frac{1}{\left\|u_{\lambda_{n}}\right\|^{2}} \int_{\Omega_{n}(a, b)}\left|u_{\lambda_{n}}\right|^{2} d t \leq \frac{C_{0}}{C_{a}^{b}\left\|u_{\lambda_{n}}\right\|^{2}} \rightarrow 0 \quad \text { as } n \rightarrow \infty .
$$

Let $0<\varepsilon<\frac{1}{3}$. Sobolev's embedding theorem implies $\left\|\nu_{\lambda_{n}}\right\|_{2}^{2} \leq C\left\|\nu_{\lambda_{n}}\right\|^{2}=C$ and $\left|\lambda_{n}\right| \leq$ $C_{1}$. It follows from the fact that there is $a_{\varepsilon}>0$ such that $\left|H_{u}(t, u)\right|<\frac{\varepsilon}{C_{1} C}|u|$ for all $|u| \leq a_{\varepsilon}$ (see $\left.\left(\mathrm{H}_{1}\right)\right)$ that

$$
\begin{aligned}
& \int_{\Omega_{n}\left(0, a_{\varepsilon}\right)} \frac{\left(H_{u}\left(t, u_{\lambda_{n}}\right), \lambda_{n} v_{\lambda_{n}}^{+}-v_{\lambda_{n}}^{-}\right)}{\| u_{\lambda_{n}} \mid} d t \\
& \quad \leq \int_{\Omega_{n}\left(0, a_{\varepsilon}\right)} \frac{\left|H_{u}\left(t, u_{\lambda_{n}}\right)\right|}{\left|u_{\lambda_{n}}\right|}\left|v_{\lambda_{n}}\right| \cdot\left|\lambda_{n} v_{\lambda_{n}}^{+}-v_{\lambda_{n}}^{-}\right| d t
\end{aligned}
$$




$$
\begin{aligned}
& \leq \frac{\varepsilon}{C_{1} C} \int_{\Omega_{n}\left(0, a_{\varepsilon}\right)}\left|v_{\lambda_{n}}\right| \cdot\left|\lambda_{n} v_{\lambda_{n}}^{+}-v_{\lambda_{n}}^{-}\right| d t \\
& \leq \frac{\varepsilon}{C_{1} C}\left(\int_{\mathbb{R}}\left|v_{\lambda_{n}}\right|^{2} d t\right)^{1 / 2}\left(\int_{\mathbb{R}}\left|\lambda_{n} v_{\lambda_{n}}^{+}-v_{\lambda_{n}}^{-}\right|^{2} d t\right)^{1 / 2} \\
& \leq \frac{\varepsilon}{C}\left\|v_{\lambda_{n}}\right\|_{2}^{2} \leq \varepsilon
\end{aligned}
$$

for all $n$. By $\left(\mathrm{H}_{4}\right),(2.9)$, and (2.11), we can take $b_{\varepsilon} \geq r_{0}$ large so that

$$
\begin{aligned}
& \int_{\Omega_{n}\left(b_{\varepsilon}, \infty\right)} \frac{\left(H_{u}\left(t, u_{\lambda_{n}}\right), \lambda_{n} v_{\lambda_{n}}^{+}-v_{\lambda_{n}}^{-}\right)}{\| u_{\lambda_{n}} \mid} d t \\
& \quad \leq \int_{\Omega_{n}\left(b_{\varepsilon}, \infty\right)} \frac{\left|H_{u}\left(t, u_{\lambda_{n}}\right)\right|}{\left|u_{\lambda_{n}}\right|}\left|v_{\lambda_{n}}\right| \cdot\left|\lambda_{n} v_{\lambda_{n}}^{+}-v_{\lambda_{n}}^{-}\right| d t \\
& \leq\left(\int_{\Omega_{n}\left(b_{\varepsilon}, \infty\right)} \frac{\left|H_{u}\left(t, u_{\lambda_{n}}\right)\right|^{\sigma}}{\left|u_{\lambda_{n}}\right|^{\sigma}} d t\right)^{1 / \sigma}\left(\int_{\Omega_{n}\left(b_{\varepsilon}, \infty\right)}\left(\left|v_{\lambda_{n}}\right| \cdot\left|\lambda_{n} v_{\lambda_{n}}^{+}-v_{\lambda_{n}}^{-}\right|\right)^{\sigma^{\prime}} d t\right)^{1 / \sigma^{\prime}} \\
& \quad \leq\left(\int_{\Omega} c_{0} \tilde{H}\left(t, u_{\lambda_{n}}\right) d t\right)^{1 / \sigma}\left(\int_{\mathbb{R}}\left|\lambda_{n} v_{\lambda_{n}}^{+}-v_{\lambda_{n}}^{-}\right|^{\tau} d t\right)^{1 / \tau}\left(\int_{\Omega_{n}\left(b_{\varepsilon}, \infty\right)}\left|v_{\lambda_{n}}\right|^{\tau} d t\right)^{1 / \tau} \\
& \quad<\varepsilon
\end{aligned}
$$

for all $n$. Note that there is $\gamma=\gamma(\varepsilon)>0$ independent of $n$ such that $\left|H_{u}\left(t, u_{\lambda_{n}}\right)\right| \leq \gamma\left|u_{\lambda_{n}}\right|$ for $t \in \Omega_{n}\left(a_{\varepsilon}, b_{\varepsilon}\right)$. By (2.12) there is $n_{0}$ such that

$$
\begin{aligned}
& \int_{\Omega_{n}\left(a_{\varepsilon}, b_{\varepsilon}\right)} \frac{\left(H_{u}\left(t, u_{\lambda_{n}}\right), \lambda_{n} v_{\lambda_{n}}^{+}-v_{\lambda_{n}}^{-}\right)}{\left\|u_{\lambda_{n}}\right\|} d t \\
& \quad \leq \int_{\Omega_{n}\left(a_{\varepsilon}, b_{\varepsilon}\right)} \frac{\left|H_{u}\left(t, u_{\lambda_{n}}\right)\right|}{\left|u_{\lambda_{n}}\right|}\left|v_{\lambda_{n}}\right| \cdot\left|\lambda_{n} v_{\lambda_{n}}^{+}-v_{\lambda_{n}}^{-}\right| d t \\
& \quad \leq \gamma \int_{\Omega_{n}\left(a_{\varepsilon}, b_{\varepsilon}\right)}\left|v_{\lambda_{n}}\right| \cdot\left|\lambda_{n} v_{\lambda_{n}}^{+}-v_{\lambda_{n}}^{-}\right| d t \\
& \quad \leq \gamma\left(\int_{\mathbb{R}}\left|v_{\lambda_{n}}\right|^{2} d t\right)^{1 / 2}\left(\int_{\Omega_{n}\left(a_{\varepsilon}, b_{\varepsilon}\right)}\left|\lambda_{n} v_{\lambda_{n}}^{+}-v_{\lambda_{n}}^{-}\right|^{2} d t\right)^{1 / 2} \\
& \quad \leq \gamma \lambda_{n}\left\|v_{\lambda_{n}}\right\|_{2}\left(\int_{\Omega_{n}\left(a_{\varepsilon}, b_{\varepsilon}\right)}\left|v_{\lambda_{n}}\right|^{2} d t\right)^{1 / 2}<\varepsilon
\end{aligned}
$$

for all $n \geq n_{0}$. Therefore, the combination of (2.13)-(2.15) implies that for $n \geq n_{0}$, we have

$$
\int_{\mathbb{R}} \frac{\left(H_{u}\left(t, u_{\lambda_{n}}\right), \lambda_{n} v_{\lambda_{n}}^{+}-v_{\lambda_{n}}^{-}\right)}{\left\|u_{\lambda_{n}}\right\|} d t<3 \varepsilon<1
$$

which contradicts with (2.8). Thus $\left\{u_{\lambda_{n}}\right\}$ is bounded.

Lemma 2.6 If $\left\{u_{\lambda_{n}}\right\}$ is the sequence obtained in Lemma 2.5, then it is also a (PS) sequence for $\Phi$ satisfying

$$
\lim _{n \rightarrow \infty} \Phi^{\prime}\left(u_{\lambda_{n}}\right)=0, \quad \lim _{n \rightarrow \infty} \Phi\left(u_{\lambda_{n}}\right) \leq \sup _{\bar{Q}} \Phi .
$$


Proof Note that $u_{\lambda_{n}}$ is bounded. From

$$
\lim _{n \rightarrow \infty} \Phi\left(u_{\lambda_{n}}\right)=\lim _{n \rightarrow \infty}\left[\Phi_{\lambda_{n}}\left(u_{\lambda_{n}}\right)+\left(\lambda_{n}-1\right)\left(\frac{1}{2}\left\|u_{\lambda_{n}}^{-}\right\|^{2}+\int_{\mathbb{R}} H\left(t, u_{\lambda_{n}}\right) d t\right)\right]
$$

and noting that

$$
\lim _{n \rightarrow \infty} \Phi^{\prime}\left(u_{\lambda_{n}}\right) \varphi=\lim _{n \rightarrow \infty}\left[\Phi_{\lambda_{n}}^{\prime}\left(u_{\lambda_{n}}\right) \varphi+\left(\lambda_{n}-1\right)\left(\left\langle u_{\lambda_{n}}^{-}, \varphi^{-}\right\rangle+\int_{\mathbb{R}}\left(H_{u}\left(t, u_{\lambda_{n}}\right), \varphi\right) d t\right)\right]
$$

uniformly in $\varphi \in W$, we obtain the conclusion.

\section{Proof of main result}

We are now in a position to prove our main result.

Proof of Theorem 1.1 Note that Lemma 2.5 implies $\left\{u_{\lambda_{n}}\right\}$ is bounded, thus $u_{\lambda_{n}} \rightarrow u$ in $W$, and $u_{\lambda_{n}} \rightarrow u$ in $L^{q}\left(\mathbb{R}, \mathbb{R}^{N}\right)$ for all $q \in[1,+\infty]$ by Lemma 2.1 , after passing to a subsequence.

By (2.3), $\Phi_{\lambda_{n}}^{\prime}\left(u_{\lambda_{n}}\right) u_{\lambda_{n}}^{+}=0$, Hölder's inequality, and Sobolev's embedding theorem,

$$
\begin{aligned}
\left\|u_{\lambda_{n}}^{+}\right\|^{2} & =\lambda \int_{\mathbb{R}}\left(H_{u}\left(t, u_{\lambda_{n}}\right), u_{\lambda_{n}}^{+}\right) d t \\
& \leq \varepsilon \int_{\mathbb{R}}\left|u_{\lambda_{n}}\right| \cdot\left|u_{\lambda_{n}}^{+}\right| d t+C_{\varepsilon} \int_{\mathbb{R}}\left|u_{\lambda_{n}}\right|^{p-1}\left|u_{\lambda_{n}}^{+}\right| d t \\
& \leq \varepsilon\left\|u_{\lambda_{n}}\right\| \cdot\left\|u_{\lambda_{n}}^{+}\right\|+C_{\varepsilon}^{\prime}\left\|u_{\lambda_{n}}\right\|_{p}^{p-1}\left\|u_{\lambda_{n}}^{+}\right\| \\
& \leq \varepsilon\left\|u_{\lambda_{n}}\right\| \cdot\left\|u_{\lambda_{n}}^{+}\right\|+C_{\varepsilon}^{\prime \prime}\left\|u_{\lambda_{n}}\right\|_{p}^{p-2}\left\|u_{\lambda_{n}}\right\| \cdot\left\|u_{\lambda_{n}}^{+}\right\| \\
& \leq \varepsilon\left\|u_{\lambda_{n}}\right\|^{2}+C_{\varepsilon}^{\prime \prime}\left\|u_{\lambda_{n}}\right\|_{p}^{p-2}\left\|u_{\lambda_{n}}\right\|^{2} .
\end{aligned}
$$

Similarly, we have

$$
\left\|u_{\lambda_{n}}^{-}\right\|^{2} \leq \varepsilon\left\|u_{\lambda_{n}}\right\|^{2}+C_{\varepsilon}^{\prime \prime}\left\|u_{\lambda_{n}}\right\|_{p}^{p-2}\left\|u_{\lambda_{n}}\right\|^{2}
$$

From (3.1) and (3.2), we get

$$
\left\|u_{\lambda_{n}}\right\|^{2} \leq 2 \varepsilon\left\|u_{\lambda_{n}}\right\|^{2}+2 C_{\varepsilon}^{\prime \prime}\left\|u_{\lambda_{n}}\right\|_{p}^{p-2}\left\|u_{\lambda_{n}}\right\|^{2}
$$

which means $\left\|u_{\lambda_{n}}\right\|_{p} \geq c$ for some constant $c$, it follows from $u_{\lambda_{n}} \rightarrow u$ in $L^{p}\left(\mathbb{R}, \mathbb{R}^{N}\right)$ that $u \neq 0$. The facts that $\Phi^{\prime}$ is weakly sequentially continuous on $W$ and $u_{\lambda_{n}} \rightarrow u$ in $W$ imply $\Phi^{\prime}(u)=0$.

Let $K:=\left\{u \in W: \Phi^{\prime}(u)=0\right\}$ be the critical set of $\Phi$ and

$$
C:=\inf \{\Phi(z): z \in K \backslash\{0\}\}
$$

For any critical point $u$ of $\Phi$, assumption $\left(\mathrm{H}_{3}\right)$ implies that

$$
\Phi(u)=\Phi(u)-\frac{1}{2} \Phi^{\prime}(u) u=\int_{\mathbb{R}}\left(\frac{1}{2}\left(H_{u}(t, u), u\right)-H(t, u)\right) d t>0 \quad \text { if } u \neq 0 .
$$


Therefore $C \geq 0$. We prove that $C>0$ and there is $u \in K$ such that $\Phi(u)=C$. Let $u_{j} \in K \backslash\{0\}$ be such that $\Phi\left(u_{j}\right) \rightarrow C$. Then the proof in Lemma 2.5 shows that $\left\{u_{j}\right\}$ is bounded, and by the concentration compactness principle discussion above we know $u_{j} \rightarrow u \in K \backslash\{0\}$. Thus

$$
\begin{aligned}
C & =\lim _{j \rightarrow \infty} \Phi\left(u_{j}\right)=\lim _{j \rightarrow \infty} \int_{\mathbb{R}}\left(\frac{1}{2}\left(H_{u}\left(t, u_{j}\right), u_{j}\right)-H\left(t, u_{j}\right)\right) d t \\
& \geq \int_{\Omega}\left(\frac{1}{2}\left(H_{u}(t, u), u\right)-H(t, u)\right) d t=\Phi(u) \geq C,
\end{aligned}
$$

where the first inequality is due to $\left(\mathrm{H}_{3}\right)$ and Fatou's lemma. So $\Phi(u)=C$ and $C>0$ because $u \neq 0$.

\section{Competing interests}

The authors declare that they have no competing interests.

\section{Authors' contributions}

All authors contributed equally to the writing of this paper. All authors read and approved the final manuscript.

\section{Author details}

${ }^{1}$ School of Mathematics and Statistics, Anyang Normal University, Anyang, Henan Province 455000, P.R. China.

²Petrochina Pipeline Company, Langfang, Hebei Province 065000, P.R. China.

\section{Acknowledgements}

Research supported by the Tianyuan Fund for Mathematics of NSFC (Grant No. 11326113) and the Key Project of Natural Science Foundation of Educational Committee of Henan Province of China (Grant No. 13A110015).

Received: 10 June 2014 Accepted: 7 August 2014 Published: 20 August 2014

\section{References}

1. Ambrosetti, A, Coti Zelati, V: Multiple homoclinic orbits for a class of conservative systems. Rend. Semin. Mat. Univ. Padova 89, 177-194 (1993)

2. Chen, G, Ma, S: Periodic solutions for Hamiltonian systems without Ambrosetti-Rabinowitz condition and spectrum J. Math. Anal. Appl. 379, 842-851 (2011)

3. Ding, Y: Existence and multiplicity results for homoclinic solutions to a class of Hamiltonian systems. Nonlinear Anal. 25, 1095-1113 (1995)

4. Izydorek, M, Janczewska, J: Homoclinic solutions for a class of second order Hamiltonian systems. J. Differ. Equ. 219, 375-389 (2005)

5. Kim, Y: Existence of periodic solutions for planar Hamiltonian systems at resonance. J. Korean Math. Soc. 48 , 1143-1152 (2011)

6. Mawhin, J, Willem, M: Critical Point Theory and Hamiltonian Systems. Applied Mathematical Sciences, vol. 74. Springer, New York (1989)

7. Omana, W, Willem, M: Homoclinic orbits for a class of Hamiltonian systems. Differ. Integral Equ. 5, 1115-1120 (1992)

8. Paturel, E: Multiple homoclinic orbits for a class of Hamiltonian systems. Calc. Var. Partial Differ. Equ. 12, 117-143 (2001)

9. Rabinowitz, PH: Homoclinic orbits for a class of Hamiltonian systems. Proc. R. Soc. Edinb., Sect. A 114, 33-38 (1990)

10. Rabinowitz, PH, Tanaka, K: Some results on connecting orbits for a class of Hamiltonian systems. Math. Z. 206, 473-499 (1991)

11. Séré, E: Existence of infinitely many homoclinic orbits in Hamiltonian systems. Math. Z. 209, 133-160 (1992)

12. Sun, J, Chen, H, Nieto, JJ: Homoclinic solutions for a class of subquadratic second-order Hamiltonian systems. J. Math. Anal. Appl. 373, 20-29 (2011)

13. Tang, X, Xiao, L: Homoclinic solutions for non-autonomous second-order Hamiltonian systems with a coercive potential. J. Math. Anal. Appl. 351, 586-594 (2009)

14. Wan, L, Tang, C: Existence and multiplicity of homoclinic orbits for second order Hamiltonian systems without (AR) condition. Discrete Contin. Dyn. Syst., Ser. B 15, 255-271 (2011)

15. Xiao, J, Nieto, JJ: Variational approach to some damped Dirichlet nonlinear impulsive differential equations. J. Franklin Inst. 348, 369-377 (2011)

16. Zhang, P, Tang, C: Infinitely many periodic solutions for nonautonomous sublinear second-order Hamiltonian systems. Abstr. Appl. Anal. 2010, 620438 (2010). doi:10.1155/2010/620438

17. Zhang, Q, Liu, C: Infinitely many homoclinic solutions for second order Hamiltonian systems. Nonlinear Anal. 72 894-903 (2010)

18. Chen, G: Non-periodic damped vibration systems with sublinear terms at infinity: infinitely many homoclinic orbits. Nonlinear Anal. 92, 168-176 (2013)

19. Chen, G: Non-periodic damped vibration systems with asymptotically quadratic terms at infinity: infinitely many homoclinic orbits. Abstr. Appl. Anal. 2013, 937128 (2013) 
20. Wu, X, Zhang, W: Existence and multiplicity of homoclinic solutions for a class of damped vibration problems. Nonlinear Anal. 74, 4392-4398 (2011)

21. Zhang, Z, Yuan, R: Homoclinic solutions for some second-order nonautonomous systems. Nonlinear Anal. 71, 5790-5798 (2009)

22. Zhu, W: Existence of homoclinic solutions for a class of second order systems. Nonlinear Anal. 75, 2455-2463 (2012)

23. Schechter, M, Zou, W: Weak linking theorems and Schrödinger equations with critical Sobolev exponent. ESAIM Control Optim. Calc. Var. 9, 601-619 (2003)

24. Costa, DG, Magalhães, CA: A variational approach to subquadratic perturbations of elliptic systems. J. Differ. Equ. 111, 103-122 (1994)

25. Wang, J, Xu, J, Zhang, F: Homoclinic orbits for a class of Hamiltonian systems with superquadratic or asymptotically quadratic potentials. Commun. Pure Appl. Anal. 10, 269-286 (2011)

26. Willem, M: Minimax Theorems. Birkhäuser, Boston (1996)

doi:10.1186/1687-1847-2014-230

Cite this article as: Chen and Zhao: Ground state homoclinic orbits of superquadratic damped vibration systems. Advances in Difference Equations 2014 2014:230

\section{Submit your manuscript to a SpringerOpen ${ }^{\circ}$ journal and benefit from:}

- Convenient online submission

- Rigorous peer review

- Immediate publication on acceptance

- Open access: articles freely available online

- High visibility within the field

- Retaining the copyright to your article 